\title{
Network-Based Identification of New Targets for Natural Products Enables Potential Uses in Aging-Associated Disorders
}

\author{
Jiansong Fang, Haobin Cai, Yunbo Chen, Qi Wang \\ Institute of Clinical Pharmacology, Guangzhou University of Chinese Medicine, Guangzhou, China
}

Background: aging that refers the accumulation of genetic and physiology changes in cells and tissues over a lifetime has a higher risk of developing various complex diseases, such as neurodegenerative diseases, cardiovascular diseases, and cancers. Over the past several decades, natural products act as anti-aging interveners with various molecular mechanisms via extending lifespan and preventing age-related diseases.

Methods: we developed an integrated systems pharmacology infrastructure to uncover new indications for various agerelated diseases by natural products. Specifically, we incorporated high quality aging-associated human or humanorthologous genes. We reconstructed a global drug-target network of natural products by integrating both experimental and the computationally predicted drug-target interactions via the predictive network models. We further built the statistical network models for identification of new anti-aging indications of natural products through via integration of the curated aging-associated genes and drug-target network of natural products.

Results: high accuracy was achieved for the statistical network model. Furthermore, we showcased multiple predicted antiaging indications of several typical natural products (e.g. resveratrol, ellagic acid, caffeic acid, and metformin with new mechanisms-of-actions.

Conclusions: this study demonstrates a novel systems pharmacology infrastructure to identify new indications for various age-related diseases by natural products. 